\title{
Separation of non-steroidal anti-inflammatory drugs by capillary electrophoresis using non-aqueous electrolyte
}

\author{
Marianne Fillet, ${ }^{1 *}$ Isabelle Bechet, ${ }^{1}$ Lucas Fotsing, ${ }^{1}$ V. Piette ${ }^{2} \dagger$ and Jacques Crommen ${ }^{1}$ \\ ${ }^{1}$ Department of Analytical Pharmaceutical Chemistry, Institute of Pharmacy, University of Liège, CHU B-26, Avenue de I'Hopital 1, B-4000 \\ Liège 1 , Belgium \\ ${ }^{2}$ Laboratory of Drug Analysis, Institute of Public Health—Louis Pasteur, Rue J. Wytsman 14, B-1050 Bruxelles, Belgium
}

Received 28 May 1999; accepted 14 June 1999

So far, most capillary electrophoresis (CE) separations have been carried out in aqueous or, to a lesser extent, mixed aqueous-organic media. Organic solvents have mainly been used in micellar electrokinetic chromatography (MEKC) and in capillary electrochromatography (CEC), most often to improve the separation of hydrophobic compounds. Non-aqueous media extended the application range of $\mathrm{CE}$ to the analysis of compounds of poor solubility in water, and also improved the selectivity of the separation of compounds which are characterized by very similar electrophoretic mobilities in aqueous media.

The aim of the present work was to investigate the separation of non-steroidal anti-inflammatory drugs (NSAIDs: niflumic acid, flufenamic acid, piroxicam, alclofenac, flurbiprofen, indomethacin, ketoprofen, naproxen, carprofen, sulindac) in CE using completely nonaqueous systems. NSAIDs are acidic compounds characterized by low solubility in water, and some of them have similar charge densities, which makes their separation difficult in aqueous systems.

In this work, the influence of different parameters such as nature and proportion of organic solvent (methanol, acetonitrile, 2-propanol), apparent $\mathrm{pH}$ (ranging from 7 to 9) and temperature (ranging from 25 to $40^{\circ} \mathrm{C}$ ) on selectivity and migration times has been studied systematically in an uncoated fused silica capillary.

Among the three organic solvents tested, methanol was found to provide important changes in selectivity. In a completely methanolic system, the cathodic electroosmotic flow was strongly decreased in comparison to the analogous aqueous system (cf. $\mu_{\mathrm{eo}}=63 \times 10^{-5} \mathrm{~cm}^{2} /$ $\mathrm{Vs}$ in the aqueous system and $\mu_{\mathrm{eo}}=7.5 \times 10^{-5} \mathrm{~cm}^{2} / \mathrm{Vs}$ in the methanolic system), because of changes in the

\footnotetext{
*Correspondence to: M. Fillet, Department of Analytical Pharmaceutical Chemistry, Institute of Pharmacy, University of Liège, CHU B-26, Avenue de l'Hopital 1, B-4000 Liège 1, Belgium.

E-mail: Marianne.Fillet@ulg.ac.be

$\dagger$ On leave.
}

dielectric properties and the viscosity of the system. Table 1 shows several significant differences in the migration order of 10 NSAIDs (text mixture) between the two systems. The range of NSAID migration times is larger in the methanol buffer than in the aqueous buffer, so that greater differences in the mobilities of NSAIDs are achieved, particularly for the group of compounds that are not resolved in the aqueous buffer. Methanol decreases the electrophoretic mobilities of NSAIDs, but also changes selectivity depending on the chemical structure of the analyte. The same buffer containing $30 \%$ acetonitrile provides a satisfactory separation for 13 NSAIDs within $14 \mathrm{~min}$ at $25^{\circ} \mathrm{C}$. Some inversions of the migration order occurred when the concentration of acetonitrile was varied, probably by changes of solvation of the analytes.

Changes in selectivity and in the migration order of NSAIDs were also obtained by changing the apparent $\mathrm{pH}$ of the methanolic electrolyte (ranging from 7 to 9), while migration times were not very much affected. These changes could sometimes result in resolution improve-

Table 1. Migration times (min) of NSAIDs in aqueous and non-aqueous systems

\begin{tabular}{llll}
\hline \multicolumn{2}{c}{ Aqueous system } & \multicolumn{2}{c}{ Non-aqueous system } \\
\hline Alclofenac & 2.68 & Niflumic acid & 5.52 \\
Flufenamic acid & 2.73 & Flufenamic acid & 6.13 \\
Niflumic acid & 2.81 & Piroxicam & 6.87 \\
Flurbiprofen & 2.90 & Alclofenac & 7.01 \\
Naproxen & 2.95 & Flurbiprofen & 7.54 \\
Carprofen & 3.12 & Ketoprofen & 7.73 \\
Ketoprofen & 3.12 & Naproxen & 7.75 \\
Piroxicam & 3.12 & Indomethacin & 8.10 \\
Indomethacin & 3.12 & Carprofen & 8.56 \\
Sulindac & 3.23 & Sulindac & 9.03 \\
\hline
\end{tabular}

Buffer: $50 \mathrm{mM}$ ammonium acetate- $13.75 \mathrm{~mm}$ ammonia in water (1) or in methanol (2). Voltage: $-25 \mathrm{kV}$ in (1) and $+25 \mathrm{kV}$ in (2). Temperature: $25^{\circ} \mathrm{C}$; wavelength: $280 \mathrm{~nm}$; capillary: uncoated fused capillary $(44 \mathrm{~cm} ; 50 \mu \mathrm{m}$ i.d. $)$. 


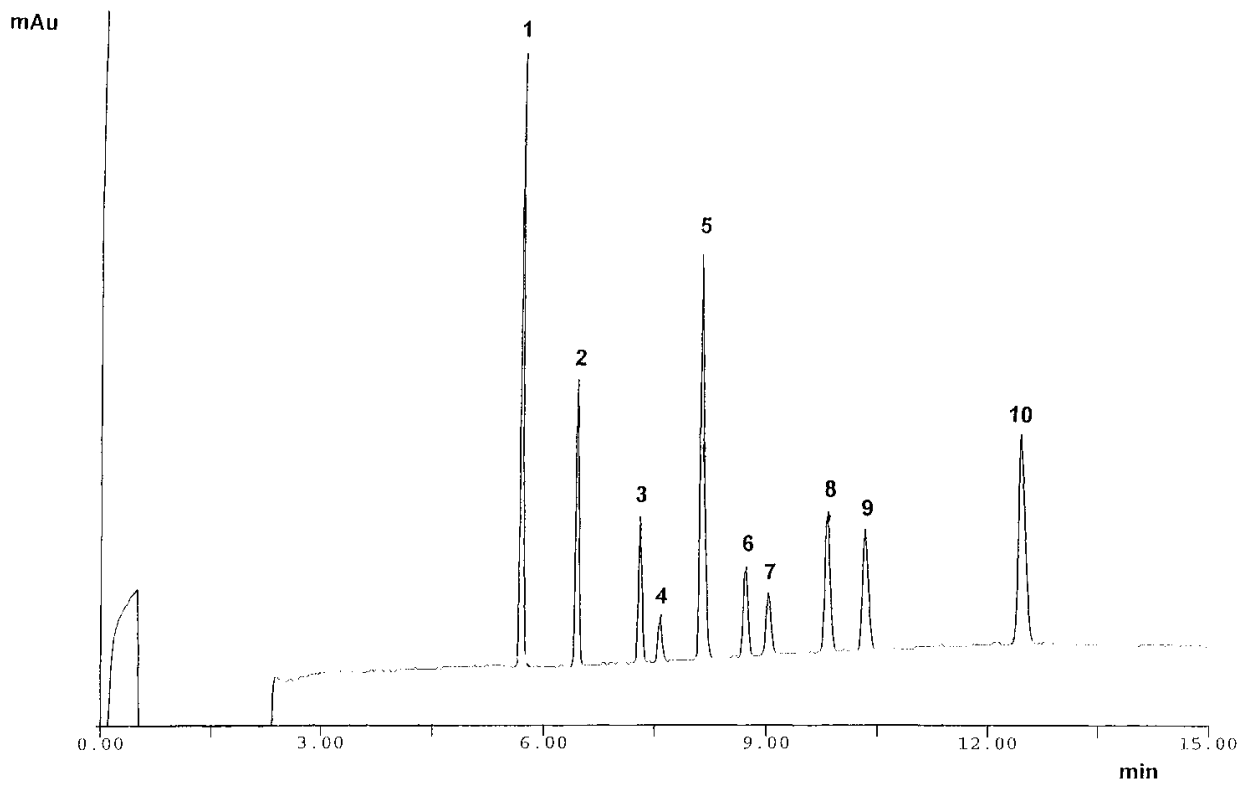

Figure 1. Separation of 10 NSAIDs in non-aqueous electrolyte. Buffer: $50 \mathrm{mM}$ ammonium acetate $35 \mathrm{mM}$ ammonia $(* \mathrm{pH} 9)$ in methanol. Temperature: $25^{\circ} \mathrm{C}$. Other conditions as described in Table 1. Peaks: 1, niflumic acid; 2, flufenamic acid; 3, piroxicam; 4, alclofenac; 5, flurbiprofen; 6, ketoprofen; 7, naproxen; 8 , indomethacin; 9, carprofen; 10, sulindac.

ment. Apparent $\mathrm{pH}$ was varied by an increasing ammonia concentration from $4.5 \mathrm{mM}(* \mathrm{pH} 8)$ to $35 \mathrm{mM}(* \mathrm{pH} 9)$. $* \mathrm{pH} 7$ and $* \mathrm{pH} 7.5$ were obtained by addition of respectively 35 and $15 \mathrm{~mm}$ of acetic acid. However, resolution modifications observed with the apparent $\mathrm{pH}$ variation do not seem to be the only consequence of this increase of ionic strength; the observed effect probably depends on the nature of the analyte, as can be concluded from inversions of migration orders. As can be seen in Fig. 1, a buffer with an apparent $\mathrm{pH}$ of 9, which corresponds to a concentration of $35 \mathrm{~mm}$ ammonia, allows for baseline resolution of the mixture of the 10 NSAIDs. High efficiency and excellent peak symmetry were found for all NSAIDs tested in the non-aqueous methanol system, in spite of a relatively wide range of migration times.

To complicate the system, three NSAIDs have been added to the test mixture (tiaprofenic acid, suprofen, indoprofen), but under the conditions $50 \mathrm{mM}$ ammonium acetate $-13.75 \mathrm{mM}$ ammonia in methanol $(* \mathrm{pH} 8.5)$ at $25^{\circ} \mathrm{C}$, some of the 13 NSAIDs coelute. An increase of temperature leads to a slight decrease of migration times for all NSAIDs, due to a decrease of the viscosity of the separation medium. Furthermore, some changes of the migration order occur as the temperature increases, resulting in improvement in resolution. The effect of temperature on the selectivity in this non-aqueous system provides an additional parameter likely to improve the separation of analytes with similar charge densities which are difficult to resolve even in non-aqueous media. However, it appears from this study that the electrophoretic behaviour of some analytes is sensitive to slight changes of temperature.

A non-aqueous electrolyte made from $50 \mathrm{~mm}$ ammonium acetate- $13.75 \mathrm{mM}$ ammonia in methanol proved to resolve $11 \mathrm{NSAIDs}$ at $25^{\circ} \mathrm{C}$ and 13 NSAIDs at $36^{\circ} \mathrm{C}$, both within $13 \mathrm{~min}$ and without a modifier besides the methanol itself. The same buffer containing 30\% acetonitrile provides a satisfactory separation for 13 NSAIDs at $25^{\circ} \mathrm{C}$.

The apparent $\mathrm{pH}$, the addition of acetonitrile and temperature are parameters which can be optimized for improvement of selectivity in non-aqueous systems. Not all effects of changes in absolute and relative migration times (selectivity) observed in the reported experiments can be explained. The usage of non-aqueous electrolytes seems to be an effective means to change and increase separation selectivity in CE, especially for more hydrophobic analytes with very similar charge densities, which are often difficult to resolve in aqueous media. 